


\title{
LA VICTIMIZACIÓN EN LAS INVESTIGACIONES CON PERSONAS AFECTADAS POR LA VIOLENCIA FAMILIAR O SEXUAL
}

\author{
Research on victimization of people affected by family or \\ sexual violence
}

Olga Bardales Mendoza*

\begin{abstract}
Resumen
Este artículo, pretende reflexionar y alertar sobre el tratamiento y a las consideraciones éticas que sobre la víctima de violencia se deben tomar en cuenta en las investigaciones científicas cuando se aborda la problemática de la violencia familiar y sexual.Toma de base la experiencia personal como investigadora en violencia familiar y sexual en diversas instituciones; así como, desde la experiencia del Estado en la promoción y producción de investigaciones; y en la evaluación de diversos protocolos externos que son alcanzados para emitir recomendaciones. Se reflexionará aquí sobre los casos más relevantes de protocolos e investigaciones que nos lleve a proponer una transformación en el rol del investigador, tendiente a asumir una actitud ética para con los participantes en las investigaciones.
\end{abstract}

Palabras clave: Victimización, ética, violencia familiar y sexual.

\begin{abstract}
This article aims to reflect and alert on the treatment and ethical considerations that have the victims of violence. These should be considered in scientific research when the domestic and sexual violence issue is analyzed.

This article is based on personal experience as a researcher in family and sexual violence in several institutions, and on experience of the State in promoting and producing research and evaluating several external protocols that are reached to issue recommendations.

We will reflect on the most relevant protocols and research which lead us to propose a transformation in the researcher's role, tending to assume an ethical attitude towards the research participants.
\end{abstract}

Keywords: Victimization, ethics family and sexual violence

\footnotetext{
* Psicóloga. Programa Nacional Contra la Violencia Familiar y Social - Ministerio de la Mujer y Poblaciones Vulnerables.obardales@hotmail.com
} 


\section{INTRODUCCIÓN}

La investigación es una práctica que se supone vital para el avance y desarrollo de las sociedades; nos permite conocer y comprender una realidad, sea cual esta fuera; es decir, nos dota de información relevante para solucionar problemas concretos en la sociedad. Pero además debemos tener en cuenta que la investigación no es sencilla y menos asequible económicamente; la metodología científica se adentra en temas de investigación complejas y trabaja con seres humanos muchas veces disminuidos en sus derechos y en su autonomía, lo que hace más difícil su accionar, y nos lleva a reflexionar sobre la aproximación que el investigador debe tener sobre dicha realidad, en este caso la violencia familiar y sexual. sin generarles mayores daños o exponerlos a riesgos a las personas que participan.

Esto es un gran reto para la comunidad científica, que debe tener claro que prepararse sólo en técnicas metodológicas científicas no es suficiente, sino que requieren tener una preparación para lograr el tratamiento ético y humano en los proyectos de investigación, porque definitivamente está comprobado que esto genera mayor validez en los resultados, en comparación a otros estudios que no consideran las pautas éticas.

Por ello, este artículo pretende tratar sobre las pautas éticas para minimizar la revictimización en las investigaciones sobre violencia familiar y sexual, considerando que las principales víctimas son las mujeres y los niños, además porque consideramos que esta problemática es un tema vigente e importante en la agenda política del país, y porque es insensato pensar que alguna de nuestras intervenciones $o$ contacto realizado a través de las investigaciones con las personas víctimas no les afecta en lo mínimo.

Investigar con seres humanos, y especialmente con víctimas trae consigo siempre un contacto con la experiencia emocional personal y privada de la persona, que conlleva muchas veces a situaciones de revictimización, que empeora la situación de la persona, si no se toma conciencia de este hecho o no se prevén estrategias para minimizarlo.

\section{LA SITUACIÓN DE LA VIOLENCIA FAMILIAR Y SEXUAL EN EL PERÚ}

La violencia en el contexto de la familia cada vez muestra índices más elevados en las estadísticas de nuestro país, hecho que genera una demanda e interés social, político y académico por tener una mejor comprensión de esta problemática, llevando a formular acciones, entre ellas las investigaciones que permita resolver y enfrentar estas situaciones que vulneran los derechos fundamentales de las personas involucradas en los hechos violentos.

De acuerdo a las últimas estadísticas proporcionadas por el INEI (2011), en su Encuesta Demográfica y de Salud Familiar (ENDES), la violencia es aún un problema vigente y grave, como se aprecia en el gráfico 1, la violencia desde el año 2000, se ha mantenido casi estable.

Gráfico 1: Violencia física y sexual contra las mujeres 15-49 años (Perú)

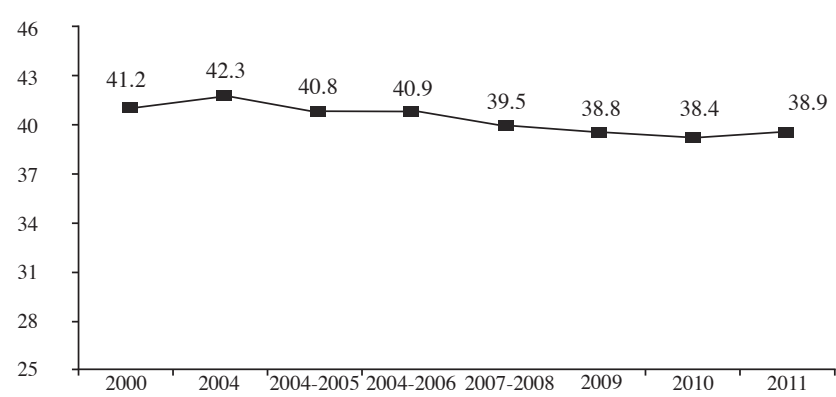

Fuente: ENDES, INEI

\section{EL TRATAMIENTO ÉTICO EN LAS INVESTIGACIONES EN VIOLENCIA FAMILIAR Y SEXUAL}

En relación al tratamiento ético, quiero partir de un diagnóstico realizado en base a una convocatoria que se realizó en el año 2010, el Ministerio de la Mujer y Poblaciones Vulnerables (MIMP), a través del Concurso Nacional de Investigaciones en Violencia Familiar y Sexual. En dicho concurso, se presentaron y evaluaron un total de 42 protocolos de investigación en esta problemática, y sus características y temas propuestos a estudiar se muestran a continuación (Ver tabla 1 y 2 ). 
Tabla 1: Proyectos por departamento

\begin{tabular}{lr}
\hline Departamento & \\
\hline Lima & 16 \\
Cusco & 10 \\
Junín & 2 \\
Ancash & 2 \\
Huánuco & 2 \\
Ayacucho & 2 \\
Arequipa & 1 \\
Ucayali & 2 \\
Tumbes & 1 \\
Puno & 1 \\
La Libertad & 1 \\
Cajamarca & 1 \\
Amazonas & 1 \\
Total & $\mathbf{4 2}$ \\
\hline
\end{tabular}

Fuente: base de datos del concurso-MIMP, 2010

Tabla 2: Proyectos por área temática

\begin{tabular}{lr}
\hline Línea Temática & \\
\hline Grupos vulnerables & 21 \\
Varones y la violencia familiar & 9 \\
Adulto mayor & 6 \\
Otros & 2 \\
Medios de comunicación & 4 \\
Total & $\mathbf{4 2}$
\end{tabular}

Fuente: base de datos del concurso-MIMMP, 2010
Estos protocolos, fueron evaluados con criterios tanto para los aspectos técnicos, pero también en las consideraciones éticas, de manera de poder tener una idea, en este último caso, de cuál es el conocimiento y la aplicación de las consideraciones éticas en los protocolos de investigación en violencia familiar y sexual por parte de los investigadores; como resultado de ello, se encontró que de los 42 proyectos evaluados, 35 fueron los que consideraron en el protocolo un acápite ético con al menos una consideración ética; mientras 7 de ellos no lo hicieron de ninguna manera.

Un análisis más cualitativo de los resultados sobre la ética, podemos apreciar en la tabla 3 y en el gráfico 1 , donde nos podemos dar cuenta que los aspectos éticos considerados por los investigadores no son uniformes; y sobre todo, el tema específico y explícito de la víctimización no es considerado en ningún protocolo, hecho que nos lleva a pensar que se requiere un trabajo de los investigadores de fortalecimiento de capacidades y sensibilización sobre este punto tan importante; y en general, sobre la violencia familiar y sexual.

Tabla 3: Proyectos según 'consideraciones éticas contempladas

\begin{tabular}{|c|c|c|c|c|c|c|c|c|}
\hline \multicolumn{1}{|c|}{ Il Concurso de Investigaciones en Violencia Familiar y Sexual - Proyectos a evaluar } \\
\hline No & $\begin{array}{c}\text { Diseño } \\
\text { participativo }\end{array}$ & $\begin{array}{c}\text { Lenguaje } \\
\text { instrumento }\end{array}$ & $\begin{array}{c}\text { Consentimiento } \\
\text { informado }\end{array}$ & $\begin{array}{c}\text { evalúa } \\
\text { riesgos }\end{array}$ & voluntario & confidencialidad & $\begin{array}{c}\text { devolución } \\
\text { resultados }\end{array}$ & $\begin{array}{c}\text { Anonimato } \\
\text { personas con } \\
\text { autonomía } \\
\text { disminuida }\end{array}$ \\
\hline Proyectos & 4 & 3 & 13 & 5 & 4 & 17 & 5 & 7 \\
\hline
\end{tabular}

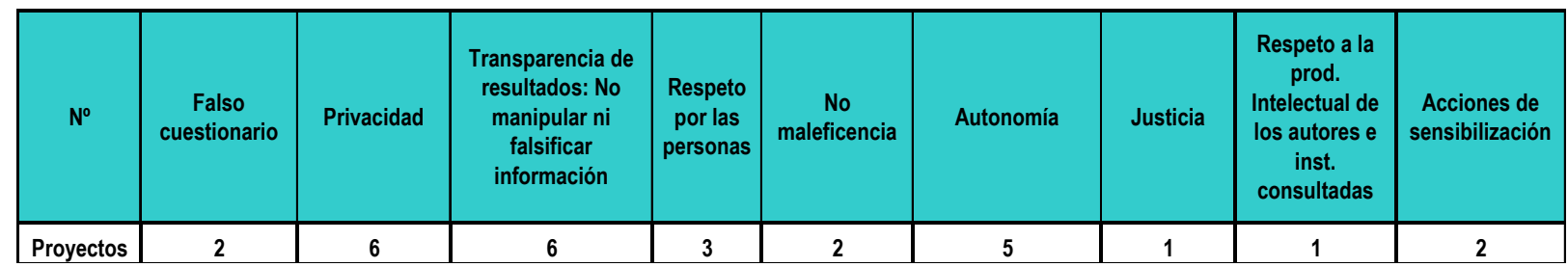

Fuente: base de datos del concurso-MIMP, 2010 


\section{Gráfico 2: Proyectos según consideraciones éticas}

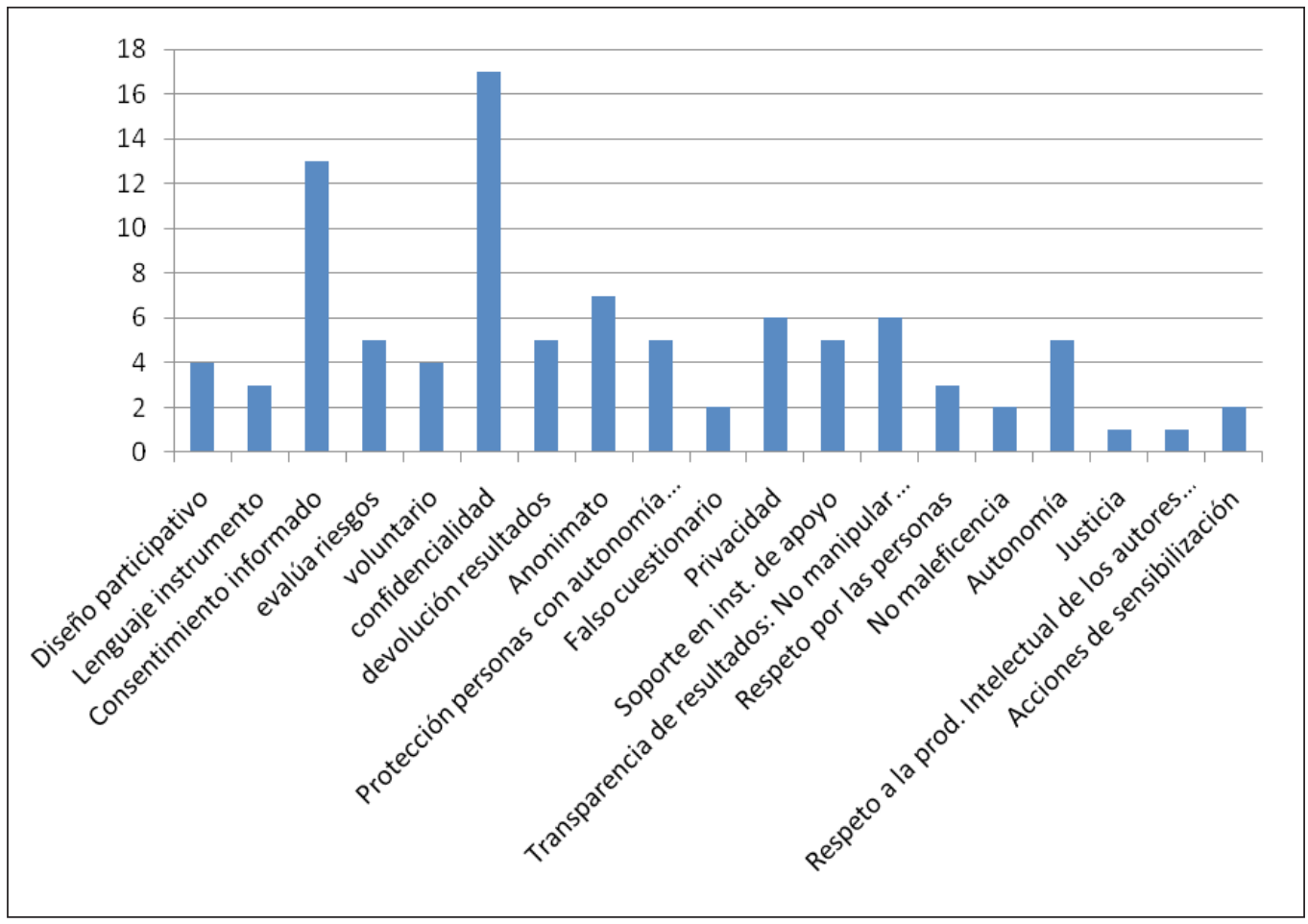

Fuente: base de datos del concurso-MIMP, 2010

\section{LA VÍCTIMIZACIÓN EN LAS INVESTIGA- CIONES EN VIOLENCIA FAMILIAR Y SEXUAL}

Primeramente, para intentar entender la relación entre la ejecución de las investigaciones en violencia familiar y sexual y la victimización; me permitiré señalar algunos conceptos importantes para lograr los objetivos de este artículo:

\section{Víctimología}

El campo de la victimología deviene del campo de la Criminología, y pudiéramos enmarcarla a mediados de la década de los cuarenta. En relación a los antecedentes más remotos de la victimología latinoamericana, tenemos:

(...) tiene sin lugar con los juristas cubanos: Diego Vicente Tejeda; Francisco Fernández; y José Ramón Figueroa quienes en el año 1929 presentaron en el Colegio de Abogados de La Habana su disertación titulada la protección a la víctima del delito, posteriormente una década después Bemjamín Mendelshon realiza estudios e investigaciones definiendo la victimología como la ciencia encargada del estudio de la víctima (Pérez, 2008: S/P).

Este mismo autor indica, que etimológicamente, la expresión victimología deriva de la palabra latina "víctima" y de la raíz griega "logos". Esta última significa, en su acepción secular, palabra discurso, estudio. Entonces, la victimología se refiere pues, en esta segunda acepción, al estudio de las víctimas del delito.

Entonces la víctimología nos da elementos para entender a la víctima, comprender su contexto, etc. Hay algunos enfoques que llevan a pensar que la víctima no está determinada por sí misma a ser una víctima, sino por el contrario, esta situación depende de factores externos que tienen que ver mucho con el desencadenamiento de esta situación; y pensando en la 
investigación científica que nos atañe en este artículo; podríamos decir, que esta es un factor externo que puede colocar a la persona víctima en una doble situación de víctima, si no se toma en cuenta previsiones.

\section{Investigaciones con las víctimas en violencia familiar y sexual y victimización}

En el contexto de la violencia contra las mujeres, el ser víctima de este hecho, ha sido definido por diversas Convenciones Internacionales, que instan a los Estados a tomar medidas correctivas sobre este problema; así tenemos, a continuación algunas definiciones: "violencia contra la mujer, constituye una violación de los derechos humanos y las libertades fundamentales y limita total o parcialmente a la mujer el reconocimiento, goce y ejercicio de tales derechos y libertades" (OEA, 1994). Esta definición tiene que ver con ser víctima en el contexto de la violación o con el no ejercicio de sus derechos humanos fundamentales, afectando esto su integridad física, psicológica o sexual como persona.

Otra definición es la dada a través de la Plataforma Beijing, que señala:

La expresión "violencia contra la mujer" se refiere a todo acto de violencia basado en el género que tiene como resultado posible o real un daño físico, sexual o psicológico, incluidas las amenazas, la coerción o la privación arbitraria de la libertad, ya sea que ocurra en la vida pública o en la privada.

Por consiguiente, la violencia contra la mujer puede tener, entre otras, las siguientes formas:

a) La violencia física, sexual y psicológica en la familia, incluidos los golpes, el abuso sexual de las niñas en el hogar, la violencia relacionada con la dote, la violación por el marido, la mutilación genital y otras prácticas tradicionales que atentan contra la mujer, la violencia ejercida por personas distintas del marido y la violencia relacionada con la explotación;

b) La violencia física, sexual y psicológica al nivel de la comunidad en general, incluidas las violaciones, los abusos sexuales, el hostigamiento y la intimidación sexuales en el trabajo, en instituciones educacionales y en otros ámbitos, la trata de mujeres y la prostitución forzada;

c) La violencia física, sexual y psicológica perpetrada o tolerada por el Estado, dondequiera que ocurra. (Naciones Unidas, 1995: punto 113)

Entonces, ser víctima de la violencia familiar o sexual, es un evento concreto y directo dirigido a una persona que la afecta en sus derechos e integridad personal, situación dada en un momento y contexto determinado. Sin embargo, debemos reconocer que frente a una víctima, en la sociedad se movilizan una serie de mecanismos institucionales generados por el Estado y la sociedad en su conjunto que tratan de resolver y corregir el daño, en la medida de lo posible. Sin embargo en esta ruta de solución del problema, tanto a nivel social, legal o psicológico, la víctima muchas veces no ve resuelto su problema; muy por el contrario, se la vuelve a revictimizar, convirtiéndose en una doble o triple víctima ya a nivel del sistema institucional y social de la sociedad donde vive.

En ese sentido, se conocen algunos tipos de victimización, entre los principales me permito mencionar:

La victimización primaria: es aquella dirigida contra la persona o el individuo particular; y La victimización secundaria: aquellos supuestos en los que la víctima ha de rememorar los hechos en virtud de los cuales ha sido victimizada. Normalmente esta victimización secundaria se produce ante la administración de justicia, los cuerpos y fuerzas de seguridad, cuando tiene que contar lo que ha sucedido (Collazos, 2007: s/p).

En este sentido, cuando el investigador científico, realiza estudios con víctimas de violencia familiar o sexual; probablemente está involucrado en situaciones de victimización secundaria frente a la persona participante en la investigación, visto que no resuelve tampoco su situación (aunque no le corresponde) y a veces la agrava, pero no hace nada tampoco para preveer o para dotar de algún beneficio directo o indirecto en los participantes. 


\section{Experiencias de investigación y la victimización}

Enfrentarse como investigador a una realidad tan sensible como la violencia familiar y sexual, requiere de un entrenamiento especial como investigador, de manera que permita que este conozca todos los aspectos implicados en dicha problemática; no solo los aspectos teóricos o legales que conlleva este problema; sino, también conocer la psicología del ser humano que sufre y que es víctima, no sólo entender el contexto que le rodea, sino los procesos psicológicos (cognitivos, afectivos y comportamentales) que operan al ser víctima de la violencia; una situación difícilmente resarcible en nuestra sociedad actual, y que muchas veces es un aspecto no resuelto probablemente durante toda la vida de estas personas afectadas.

Los investigadores cuando estudian una problemática, definen su muestra de estudio; es decir, señalan de acuerdo a sus objetivos el tipo de personas que van a participar en los estudios; aspecto que nos pone frente a un tema importante de discusión, como es el preguntarnos: ¿Cómo enfrentarnos a una persona víctima? ¿Es ella/ el totalmente autónomo para tomar decisiones y participar en una investigación?.

Respuestas que interesan responder en este artículo, que trata de cómo se debe estudiar y enfrentar éticamente a las personas víctimas de violencia familiar y sexual en las investigaciones. Las víctimas de violencia familiar y sexual son un grupo bastante demandante de acuerdo a las investigaciones realizadas en nuestro país; esto se sustenta en la sistematización realizada por Bardales, Vásquez, Ortiz, y Mendoza (2012) entre los años 2006-2010 sobre investigaciones en violencia familiar y sexual realizadas en el Perú; allí vemos como las personas víctimas son las más consideradas en los estudios (ver gráfico 3); sin embargo, la preocupación más grande es conocer si en estas investigaciones se consideran o han considerado acciones que minimicen la revictimización, acciones que pueden estar relacionadas con la confidencialidad, privacidad, beneficios, derivaciones brindadas, etc.; pero que no son explícitas en los informes de las investigaciones.

\section{Gráfico 3: Personas que participan en las muestra de las investigaciones en violencia familiar y sexual}

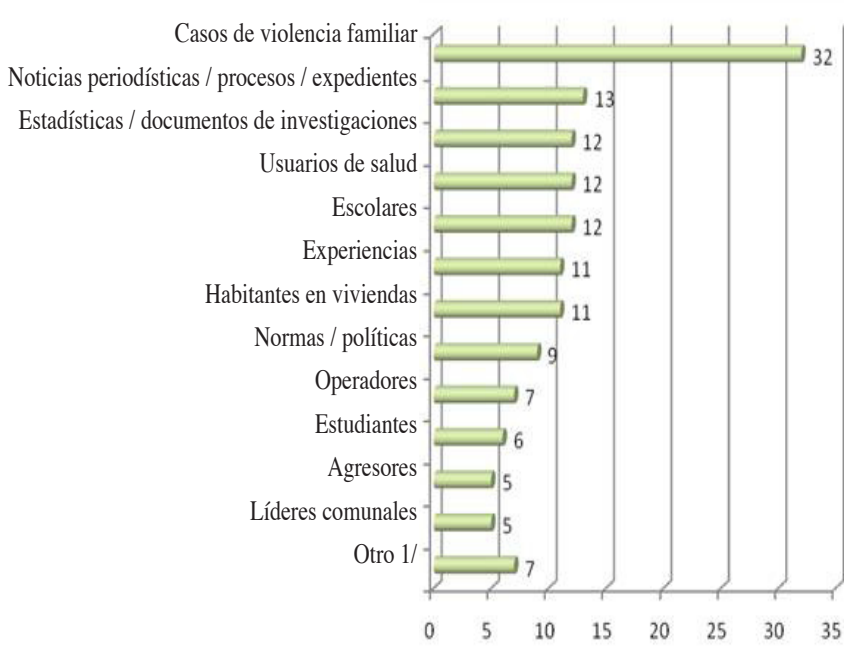

Fuente: Base de datos investigaciones 2006-2010, PNCVFS-MIMP

Lamentablemente, han sido muchos años que conocemos que diversas investigaciones están interesadas en estudiar el problema de la violencia familiar y sexual, lo cual nos parece importante; pero nunca hemos visto que en dichos protocolos o en los informes de investigación se haya contemplado el tema de la revictimización, tema que probablemente no se conoce o no se quiere reconocer, puesto que la tradición investigativa o la formación del investigador ha estado ausente hasta nuestros días una formación ética, y muchas veces se considera a la investigación valiosa como un medio sólo para obtener información, sin pensar que finalmente, la investigación es una oportunidad, a veces única, de encuentro con el otro, un proceso comunicativo entre dos personas, un investigador y un ser humano que está sufriendo una situación que va más allá de su manejo personal.

En este sentido, es importante que el investigador sea consciente de los efectos que puede generar su intervención investigativa con el fin de obtener información en la víctima (sujetos de estudio), que expone a la persona afectada por la violencia a confrontarse necesariamente con el hecho violento pasado, trayéndola a la actualidad, reviviéndola; hecho que probablemente a la fecha del estudio ha 
sido procesado emocionalmente de diversas maneras por la víctima; por ejemplo, la víctima puede estar demandante de ayuda, tener una desesperanza aprendida del sistema que la ha revictimizado por demás, puede haber generado mecanismos psicológicos de negación para olvidar esta situación dolorosa; y que ahora sin más ni más, un investigador se le acerca abogando en nombre del altruismo frente a la ciencia para que colabore con información que muchas veces la persona no sabe para qué le sirve, pero acepta porque su capacidad psicológica está disminuida y probablemente es una víctima crónica. Por ello, resulta irresponsable si no tenemos claro que con nuestra intervención investigativa estamos probablemente abriendo heridas cerradas o a medio cerrar o generando riesgos innecesarios; convirtiéndonos de esta manera victimarios institucionales.

Como sabemos, la violencia es un hecho muy doloroso que muchas veces es difícil de resolver en un sistema legal y social ineficiente; y por ello, es que la persona afectada por este hecho se le denomina víctima, pues lleva un pasivo emocional y que es difícil no afectarla si conversamos con ella o con él sobre el tema en una investigación.

En base a estas reflexiones, la pregunta es ¿podemos evitar la revictimización en los procesos de aproximación investigativa que asumen como sujetos de estudio a las víctimas de violencia familiar y sexual, considerando que probablemente en la mayoría de estos casos (por no decir, todos), el problema no ha sido resuelto, es más, el propio sistema las ha revictimizado?. La respuesta, frente a esta pregunta es no podemos evitarla, pero sí podemos minimizarla. Es decir, por ejemplo, tener un diálogo a través de una entrevista con una víctima, genera procesos cognitivos y afectivos de recordación $\mathrm{y}$ afloran sentimientos negativos que inevitablemente reviven frente a las preguntas del investigador que está frente al entrevistado víctima.

Por ello, se sostiene la necesidad de poder reflexionar y trabajar sobre el rol y las competencias que debe tener los investigadores en esta problemática, incluido la preparación especial que debe tener el personal de campo que se enfrenta directamente a las personas que participan en los estudios.
Entre las consideraciones éticas que son parte de la estrategia ética que se deben considerarse en las investigaciones que estudian a víctimas de violencia familiar, y que es parte de la experiencia personal en los estudios desarrollados sobre esta problemática, me permito señalar los siguientes:

1. El consentimiento informado: Este es un proceso muy importante, en el cual se debe aplicar antes de que la persona decida participar en el estudio; es importante considerar que este debe contener toda la información clara y necesaria que la persona debe y requiere saber para aceptar su participación y conocer a qué se enfrentará en la investigación; en el consentimiento, se le debe señalar que probablemente el proceso de diálogo de la investigación puede generarle alguna incomodidad en la recordación de hechos de violencia pasados; que el diálogo no tiene ningún fin terapéutico o es parte de este, sino que se pretende conocer su experiencia con la violencia para contribuir a la ciencia. De otro lado, también se puede señalar, que si bien no es parte del objetivo del estudio, pero probablemente el diálogo también puede generarle un sentimiento positivo de desahogo y confort en el hecho de que alguien la escucha y se interesa en su situación, lo que puede significar un proceso curativo.

2. Selección y capacitación cuidadosa del equipo de campo: El perfil del personal de campo que está enfrente de la persona, debe cumplir con requisitos mínimos; entre ellos, contar con cierta preparación profesional que permita manejar algunas técnicas de manejo del diálogo y de la comunicación eficaz, empatía y manejo de emociones. Además, de actitudes de respeto, justicia, humanística frente al otro; trabajo personal, y finalmente, conocimiento de la problemática de la violencia familiar y sexual.

3. Instrumentos que minimicen la victimización: Es complicado entendiendo que si vamos a estudiar la violencia como experiencia, no vayamos a realizar preguntas sobre este hecho que genere procesos de recordación dolorosos; sin embargo, algunas pautas son importantes, como por ejemplo: si los datos de la violencia, lo podemos recoger de otra fuente que no sea la víctima, lo deberíamos hacer (por ejemplo, registros administrativos de servicios); no hacer preguntas similares o repetitivas sobre la violencia; 
dejar que la persona en un proceso personal señale el camino y la dinámica de las preguntas, sobre todo en los estudios cualitativos. En los estudios cuantitativos, evitar usar algunas palabras fuertes o estigmatizantes que se refieran a la violencia o a la persona afectada; así como, evitar connotaciones de juicios de valor.

4. Coordinar con redes de recuperación: Por un tema de beneficencia y justicia; es una responsabilidad del equipo de investigación previamente al estudio coordinar con los servicios locales que brindan atención o recuperación en los casos de violencia familiar y sexual, de manera de tener la posibilidad de derivar $\mathrm{u}$ ofrecer a la víctima que participa en el estudio de una posibilidad de insertarse en un proceso de recuperación, que probablemente sin la oportunidad de la investigación, no habría podido acceder.

En ese sentido la investigación es una oportunidad valiosa para la persona estudiada, que muchas veces no se la percibe.

Entonces, estas son algunas acciones que debemos tener en cuenta desde las investigaciones, cuando se aborda esta problemática con víctimas. Esta situación nos lleva a reflexionar y a generar procesos de cambio y redefinición del rol de la investigación científica; es decir una investigación más ética, más humana; una investigación que no busque únicamente el dato, sino que busque la validez del dato con sentido humano y respeto a los derechos.

La investigación en esta problemática, con personas afectadas (víctimas u otros), no debe ser vista como imposible y menos complicada, o pensar que los aspectos éticos son un obstáculo para la investigación. De ninguna manera, la ética es la que nos llevará a salvar a la humanidad; y desde la investigación a comprender las realidades.

La investigación debe ser vista como una oportunidad valiosa, no sólo para conocer la realidad que nos interesa estudiar desde el lado científico; sino para alcanzar algún beneficio y justicia científica a un grupo de personas directamente afectadas por la violencia, y que muchas veces no han sido escuchadas o están siendo revictimizadas reiteradas veces por el sistema social.
Una investigación importante que me permito mencionar en relación al tratamiento de la victimización, es un estudio realizado en el año 2009 por los investigadores del Programa Nacional contra la Violencia Familiar y Sexual (PNCVFS) del Ministerio de la Mujer y Poblaciones Vulnerables; este estudio se desarrolló con víctimas directas de tentativa de feminicidio y con víctimas indirectas; es decir, familiares de mujeres asesinadas, víctimas no presentes entre nosotros. Primero, como sabemos es imposible no victimizar, porque en el proceso investigativo requerimos dialogar sobre los hechos dolorosos con los participantes; sin embargo, se tuvo que analizar el tratamiento ético que permitió minimizar la revictimización; entre estos, se consideró importante el momento en que podíamos entrar a intervenir desde la investigación con los familiares de las mujeres asesinadas. Un primer punto que se reflexionó, es el proceso de luto de los familiares, el proceso de reacomodación psicológica de la dinámica familiar, considerando esta pérdida; y finalmente, la necesidad que la familia esté insertada en un servicio de atención de la violencia, en este caso los Centros Emergencia Mujer (CEM).

Estos tres aspectos mencionados, fueron importantes para minimizar cualquier victimización de parte de la investigación; en realidad hay poca información teórica que nos diga cuando culminan los procesos de luto o pérdida; o criterios claros de reacomodación psicológica familiar o sobre la obligatoriedad de que los casos están insertados en algún servicio de violencia; pero para efectos de nuestro estudio, se establecieron los siguientes criterios, antes de enfrentarnos en el proceso investigativo con las víctimas:

- Haber pasado como mínimo dos meses después de ocurrido el hecho fatal (después del shock, rabia y con deseos de hacer algo para resarcir dicho daño). Este momento fue evaluado por los profesionales del CEM, quienes nos brindaron la información del estado actual de los deudos.

- La reacomodación psicológica de la dinámica familiar, se operacionalizó, cuando los familiares reciben terapia psicológica y cuando se han desplegado acciones de tipo social como 
evaluación social familiar, colocación de hijos con familiares o instituciones (dependiendo del caso), entre otros.

- Estar insertados en el CEM, servicio que les proporcionó apoyo tanto legal, social como psicológico, necesario para la recuperación familiar.

En ese sentido, estas pautas éticas nos permitieron aproximarnos a los deudos con mayor seguridad de que la revíctimización estaba minimizada y que detrás de nuestra intervención habían servicios que estaban trabajando los procesos dolorosos de estas personas.

\section{CONCLUSIONES}

- Las investigaciones con seres humanos y especialmente con temas delicados como la violencia familiar y sexual, requieren un tratamiento ético considerado en los protocolos e informes de investigación.

- En las investigaciones con víctimas de violencia familiar o sexual, es imposible que, en el proceso de diálogo o recordación de los hechos no se revic1timice; sin embargo, es posible minimizar este hecho teniendo en cuenta las consideraciones éticas anteriormente mencionadas.

- Estudiar a las víctimas de violencia familiar y sexual, requiere un conocimiento sobre la problemática a nivel psicológico, legal y social.

- Es necesario que los procesos de formación en las universidades sobre investigación incorporen en sus currículos los aspectos éticos.
- Es necesario plantear procesos de selección y formación de capacidades especiales para el personal de campo, de manera que se aproximen en el proceso investigativo con actitud humanista frente a las personas afectadas por la violencia familiar y sexual.

\section{REFERENCIAS}

Bardales, Vásquez, Ortiz, y Mendoza (2012). Estado de las investigaciones en violencia familiar y sexual. 2006-2010. MIMP.

Collazos, Marisol (2007). Victimología. Curso 2006/07 Licenciatura en Criminología. Universidad de Murcia, España. Universidad de Murcia. Consultado el 10 de noviembre, de http://www. marisolcollazos.es/victimologia/VictimologiaIntroducción.html

Instituto Nacional de Estadística e Informática-INEI (2011). Informe de La Encuesta Nacional de Demografía y Salud Familiar-ENDES. Lima: INEI.

Naciones Unidas (1995). Declaración y Plataforma de Acción de Beijing. La Cuarta Conferencia Mundial sobre la Mujer. N.U.

Organización de Estados Americanos - OEA (1994). Convención Interamericana para Prevenir Sancionar y Erradicar la Violencia contra la Mujer. Belem do Pará: OEA

Pérez, C. (2008). La Victimización desde el fenómeno de la violencia. Consultado el 12 de mayo 2012, de http://www.articuloz.com/noticias-y-sociedadarticulos/la-victimizacion-desde-el-fenomeno-dela-violencia-390514.html.

Fecha de recepción: 16 de octubre, 2012

Fecha de aceptación: 5 de noviembre, 2012 